\title{
A New Coalition Formation Approach for Power Losses Reduction in Electrical Power Micro-Grids
}

\author{
E. F. Kelash ${ }^{a, *}$, Osama Abdel-Raouf ${ }^{b}$, Hassan N.A. Ismail a and M. A. Elsisy ${ }^{a}$ \\ ${ }^{a}$ Basic Engineering Sciences Department, Benha Faculty of Engineering, Benha University, \\ Benha, Egypt \\ b Operations Research and Decision Support Department, Faculty of Computers and \\ Information, Menoufia University, Menoufia, Egypt
}

\begin{abstract}
In recent years, the technology of micro-grids (MGs) has gained a lot of interest. MGs utilize distributed generators (DGs) to locally meet the needs of consumers. This decentralized nature increases the efficiency of the system in addition to improving the electrical service reliability. In this paper, coalitional game theory is used to study the process of local power exchange among a set of MGs. A coalition forming (CF) algorithm which depends on the topology of the network is proposed. The presented algorithm is scalable i.e. can capture a substantial number of MGs which makes it applicable in real systems. Besides the macro-station (MS), each formed coalition comprises a set of MGs with a lack of power to purchase and a set of MGs that have an excess of power to sell. Within each coalition, quadratic programming $(Q P)$ is used to organize the transition of power among MGs and MS so as to reduce the power losses. To illustrate the significant impact of the presented procedure, a full numerical example is introduced. The analysis and simulation show that the proposed algorithm results in a decrease in the average power losses per bus, reaching up to $37.6 \%$ enhancement compared with the non-cooperative situation.
\end{abstract}

Keywords-Game theory; Coalition Formation; Micro-Grid; Smart-Grid; Power Losses; Quadratic Programming

\section{INTRODUCTION}

In the traditional power system illustrated in Figure 1, the generation tends to be centralized. The power is generated at central generation stations, transmitted over transmission lines (TLs) until it reaches the consumers at distribution regions [1,2]. This centralized nature of the current power system has a lot of drawbacks, including: 1) Any fault in the main grid can harm a wide area of the system. 2) Due to the long distance between the generation stations and the consumers which can reach thousands of miles, a huge amount of the power transmitted is dissipated over TLs and in the transformers within the network. 3) The main grid takes full responsibility for meeting all the power needs of the consumers, which makes it suffer in peak load times. On the other hand, the MG technology depends on DGs as sources to locally meet the needs of consumers. It is introduced as a promising technology to overcome the drawbacks of the current system [3]. The MGs have the ability to enhance the network performance besides the reduction of system operation costs.

MGs are electrical subsystems that consists of a group of renewable energy DGs such as solar cells, wind turbines and fuel cells connected to a group of local loads (domestic, industrial and commercial) and equipped with storage devices (batteries, fly wheels and storage capacitors) [4]. MGs operate at medium and low voltage levels which facilitate the operation of power exchange. Depending on the operation conditions, the MG systems can operate either connected to or disconnected from the main grid [5]. The electrical part which is responsible for this switching process is the coupling switch. Smart meters and communication systems have a significant rule in the proper operation of MG networks. As shown in Figure 2, each MG is equipped with a set of smart meters in addition to a MG control center (MGCC) to 
exchange the information about generation and consumption levels with other MGs and with the distribution MS. The MGCC is introduced in [6] as a multi-task controller. It can be used in optimizing and coordinating the operation of MGs. The MS has a power management center (PMC) which acts as a master to coordinate the power transfer among the MGs connected to this MS. The PMC collects the data of each MG through the MGCCs periodically. Depending on the data collected, the process of CF and power coordination is conducted. The implementation of MGs in the distribution level has a lot of advantages from both the grid and the user perspective. The authors of $[4,7,8]$ indicate the advantages of MGs, such as 1) Enhancing the quality of power. 2) Reducing carbon emissions as it depends on green energy sources. 3) Reducing the power losses incurred in TLs. 4) Mitigate the load on the main grid in peak times. 5) Postpone updating the system as it associates with the main grid in meeting the needs of the connected loads.

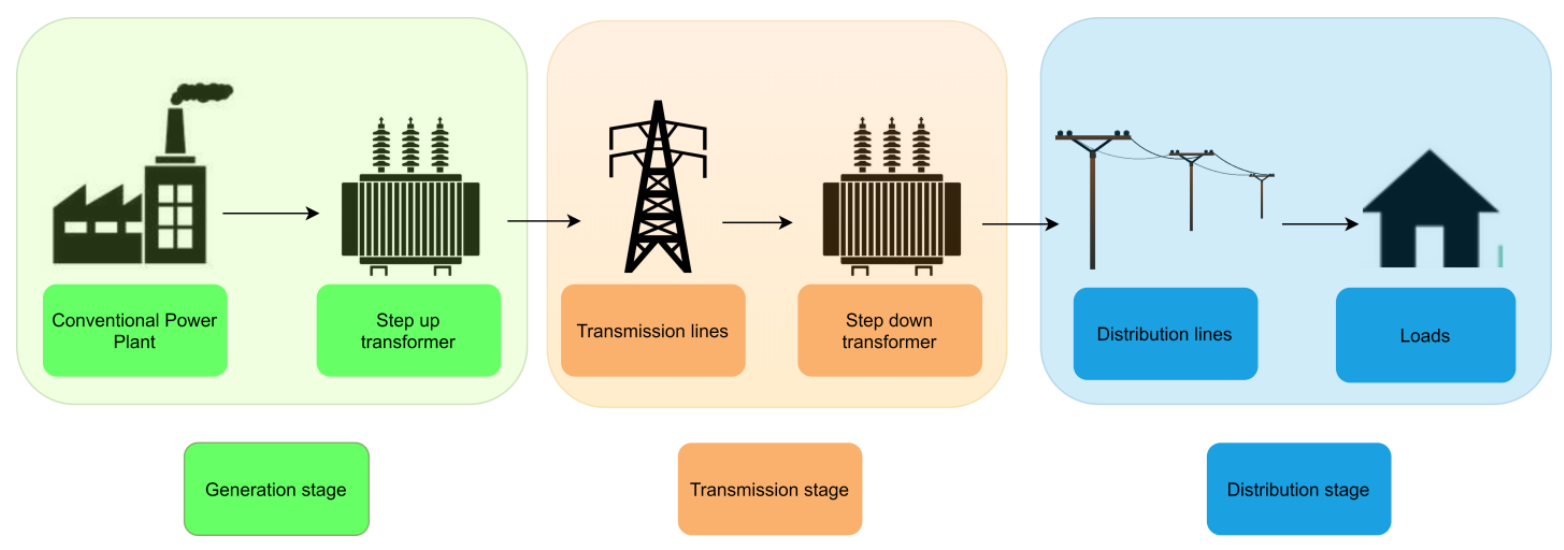

Figure 1. Traditional power system from generation to consumer.

Despite all advantages of MG technology, it still facing a lot of obstacles on many levels, such as protection against faults, synchronization with the main grid, control of power fluctuations of renewable energy sources and implementation in real systems [8]. Thus, the research areas in MG technology involve control systems, protection techniques, MG architecture, and optimization and decision making studies. The attention of this article is focused on applying the concepts of cooperative game theory in local power transmission among MGs.

Game theory (GT) is a mathematical tool which is dedicated to solve decision making situations among a number of rational players [9]. GT is divided due to the behavior of players into two branches: a) Cooperative GT and b) Non-cooperative GT. The authors of $[2,10,11]$ review the applications of GT in MG systems. The work carried out by the authors of [12] presents a CF game among a number of MGs. The authors focus on the generation of the coalition structure more than optimal matching within each coalition. The results of [12] show an improvement in the power losses in the case of the proposed algorithm reaching $31 \%$. A key limitation of this research is that the merge and split algorithm used in this work is NP hard which makes it not scalable, thus it can't capture a large number of stations [13]. The authors of $[14,15]$ study the same problem. The algorithm used in [15] is a heuristic algorithm which makes the numerical calculations more simpler. The major drawback of the work done in [15] is that the circuit model used is not realistic as the authors assumed that the medium voltage equals the low voltage value, in addition to that, the numerical data don't reflect the problem in a proper way. The authors of [15] concluded that as the number of MGs increases, the power dissipation decreases, but the results show that with $2 \mathrm{MGs}$ the improvement is $46.95 \%$ and with $10 \mathrm{MGs}$ the improvement is $40.11 \%$. The authors of [14] uses a price scheme to reflect the effect of cooperation in decreasing the cost of power in terms of power dissipation. Shapley value is used in [14] to distribute the worth among each coalition members. The main problem in the work of [14] is the merge and split algorithm, in addition to the not realistic circuit model used. The authors of [13] present a hierarchical algorithm with a quadratic complexity which makes it more scalable. The model used in [13] assumes that a MG achieves benefits, if the power 
traded with the MS is reduced. Medium TL $\pi$-model is used in [16] as a representation of the connections among the MGs and between the MGs and MS which makes it more realistic. The authors of [17] uses coalitional game model combined with auction theory to improve not only the utility of each MG, but also the utility of the entire system.

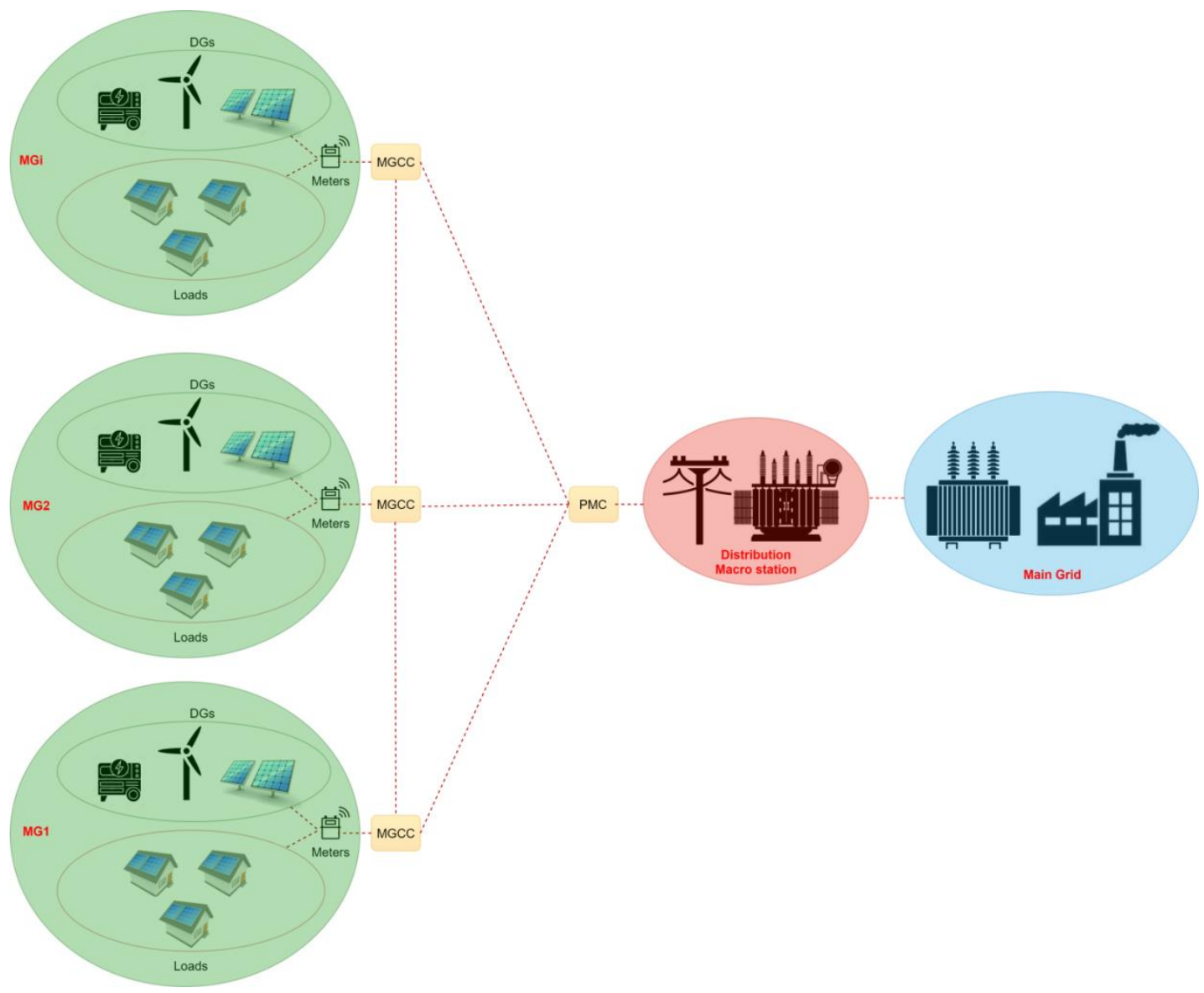

Figure 2. Architecture of MG distribution system.

Based on the system model introduced in $[12,14,15]$, the aim of this paper is to propose a novel coalition mechanism. Like the k-mean clustering, the presented algorithm is distance based with a low time complexity; therefore, it is applicable in real networks with a large number of stations. Within each coalition, the aim is to reduce the power losses in addition to properly share the power among seller MGs and buyer MGs. QP is used to match the MGs and the MS inside each coalition. Short TL model is used to represent the electrical connections among the MGs and between each MG and the MS. This model makes the problem more realistic. The impact of the cooperative behavior on the average losses per bus, overall system losses and system's transmission efficiency is discussed.

The remainder of the paper is organized as follows: In section II, the system model is presented. Section III discusses the cooperative behavior of MGs as a CF game. In section IV, the coalition structure forming algorithm and the matching problem will be explained. Section V summarizes the simulation results. Finally, section VI draws the conclusions and future work. 


\section{SYSTEM MODEL}

The distribution sector of the modified power system consists of a set of distribution MSs. Each MS is connected from the medium voltage side to the main grid and from the low voltage side to a set $\mathrm{N}$ of MGs as shown in Figure 2. Due to the nature of renewable energy sources, the amount of generated power $\left(\mathrm{P}_{\mathrm{G}}\right)$ differs from an hour to another throughout the day. The behavior of the consumers connected to each MG determines the amount of power $\left(\mathrm{P}_{\mathrm{D}}\right)$ needed to feed the loads at a certain time frame. Hence, the net power of $\mu G_{i} \in N$ could be represented by the quantity $P_{\text {net }}=P_{G_{i}}-P_{D_{i}}$. The value of $P_{n} t_{i}$ determines the nature of each MG. $\mu G_{i}$ acts as a seller, if it has a surplus of power $\left(P_{n^{n} t_{i}}>0\right)$, it behaves as a buyer, if it has a deficit of power $\left(\mathrm{P}_{n e t_{i}}<0\right)$, or it can stand alone, if $P_{n e t_{i}}=0$ [12].

MGs with excess of power and MGs with lack of power needs to sell or purchase power to or from other stations (MGs or the MS). This power exchange process causes an amount of power to be dissipated in TLs between the seller and the buyer.

As the distance between the stations within the distribution network tends to be small (less than 80 $\mathrm{km}$ ), the TL between any 2 stations (MG-MG or MG-MS) could be represented as a short distance TL. In short TLs, shunt admittance could be neglected [18]. The equivalent circuit of short distance TL is shown in Figure 3.

If there is no coordination between the MGs, all MGs do not have any choice but to exchange their power $\mathrm{P}_{\text {net }}$ with the main grid via the MS. This case is called the non-cooperative case and the power is transmitted over medium voltage level. This process of power transfer causes an amount of power to be dissipated in TLs and power transformer connecting the MG to the MS. The amount of dissipated power between $\mu G_{i}$ and the MS (neglecting the transformer losses) is formulated by [19] as $\mathrm{P}_{\mathrm{i}-\mathrm{MS}}^{\text {losses }}=3 \mathrm{I}^{2} \mathrm{R}$, where $\mathrm{R}$ is the line resistance in ohms and the magnitude of the current $\mathrm{I}$ is calculated as $|\mathrm{I}|=$

$\frac{P_{\text {flow }}}{\sqrt{3} V_{s} \cos \varphi_{s}}$ s.t. $P_{\text {flow }}$ is the amount of power flowing in the line, $V_{s}$ and $\cos \varphi_{s}$ represent the sending end voltage and power factor, respectively. Thus,

$$
\mathrm{P}_{\mathrm{i}-\mathrm{MS}}^{\text {losses }}=\frac{\mathrm{R}}{\left|\mathrm{V}_{\mathrm{s}}\right|^{2} \cos ^{2} \varphi_{\mathrm{s}}}\left(\mathrm{P}_{\text {flow }}^{2}\right),
$$

If the amount of power dissipated in the transformer is taken into account, then the amount of losses could be calculated as:

$$
\mathrm{P}_{\mathrm{i}-\mathrm{MS}}^{\text {losses }}=\frac{\mathrm{R}}{\left|\mathrm{V}_{\mathrm{s}}\right|^{2} \cos ^{2} \varphi_{\mathrm{s}}}\left(\mathrm{P}_{\text {flow }}^{2}\right)+\beta \mathrm{P}_{\text {flow }},
$$

where $\beta$ represents the fraction of power dissipated in the transformer. The value of $\mathrm{P}_{\text {flow }}$ in every line depends on the amount of net power $P_{\text {net }}$ of each MG. The amount of the flowing power between $\mu G_{i}$ and the MS is given by

$$
P_{\text {flow }(i)}= \begin{cases}P_{\text {net }_{i}} & \text { if } \mathrm{P}_{\text {net }_{i}}>0 . \\ \mathrm{X}_{\mathrm{i}}^{*} & {\text { if } \mathrm{P}_{\text {net }}<0 .}_{\mathrm{i}}<0 \\ 0 & \text { otherwise. }\end{cases}
$$

where $\mathrm{x}_{\mathrm{i}}^{*}$ is the amount of power that should be provided by the seller (MS) to meet the requirements of the buyer $(\mathrm{MG})$ having a deficit of power $\left(\mathrm{P}_{\text {net }_{\mathrm{i}}}<0\right)$. The authors of [12] defines the value of $\mathrm{x}_{\mathrm{i}}^{*}$ to be a root of the equation:

$$
x_{i}=P_{i-M S}^{\text {losses }}+p_{i}^{r e q}=\frac{x_{i}^{2} R_{i-M S}}{\left|V_{s}\right|^{2} \cos ^{2} \varphi_{s}}+\beta x_{i}-P_{n e t}{ }_{i}
$$


where $p_{i}^{r e q}=-P_{n^{2} t_{i}}, P_{n^{2} t_{i}}<0$ and $R_{i-M S}$ is the resistance of the TL linking $\mu G_{i}$ and the MS. Equation (4) could be rewritten as the quadratic equation:

$$
\mathrm{x}_{\mathrm{i}}^{2}+\frac{\beta-1}{\mathrm{R}_{\mathrm{i}-\mathrm{MS}}}\left(\left|\mathrm{V}_{\mathrm{S}}\right|^{2} \cos ^{2} \varphi_{\mathrm{s}}\right) \mathrm{x}_{\mathrm{i}}-\frac{\left|\mathrm{V}_{\mathrm{s}}\right|^{2} \cos ^{2} \varphi_{\mathrm{s}}}{\mathrm{R}_{\mathrm{i}-\mathrm{MS}}} \mathrm{P}_{\text {net }_{\mathrm{i}}}=0,
$$

By solving equation (5) and depending on the values of $R_{i-M S}, \beta, P_{n e t}$ and $\cos \varphi_{s}$, the equation can submit zero, one or two solutions. In the case of one solution, the equation has two repeated roots, therefore $\mathrm{x}_{\mathrm{i}}^{*}$ directly equals this root. When the equation has two distinct roots, the smallest root is chosen to equal $\mathrm{x}_{\mathrm{i}}^{*}$ in order to reduce the amount of power losses. In the last case, the equation has no solution. This means that the MS has no ability to generate the amount of power needed by $\mu G_{i}$. In this case, the MS sends the maximum amount of power it can provide; therefore $x_{i}^{*}=\frac{(1-\beta) *\left|V_{S}\right|^{2} \cos ^{2} \varphi_{s}}{2 R_{i-M S}}$.

Equation (2) emphasizes that the amount of power losses is reliant on several quantities. These are: the amount of power $\mathrm{P}_{\text {flow }}$ to be traded between the MG and the MS, the Euclidean distance between the MG and the MS represented by the resistance of the line $\mathrm{R}_{\mathrm{i}-\mathrm{MS}}$, the power factor $\cos \varphi_{\mathrm{S}}$ and the voltage level $\mathrm{V}_{\mathrm{S}}$ under which the power is transmitted.

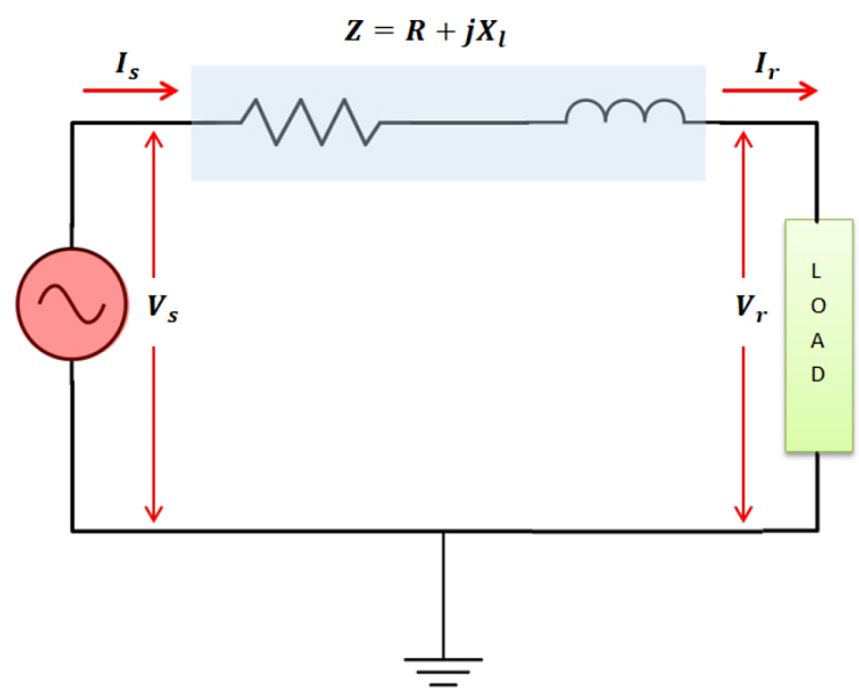

Figure 3. Short TL equivalent circuit.

The aim of the problem is to maximize the payoff of each MG by minimizing the amount of power lost in the transmission system. Thus, the utility function of any $\mu G_{i}$ is calculated as follows:

$$
\mathrm{u}\left(\left\{\mu \mathrm{G}_{\mathrm{i}}\right\}\right)=-\alpha_{\mathrm{i}} \mathrm{P}_{\mathrm{i}-\mathrm{MS}}^{\text {losses }},
$$

where $\alpha_{i}$ is the unit price of each megawatt of power lost in the TL between $\mu G_{i}$ and the MS.

\section{COALITION GAME FOR MG LOCAL POWER TRADE}

In the non-cooperative case, the MGs trade all power directly with the main grid through the MS. This process has a lot of disadvantages, including: 1) A considerable amount of the transmitted power is dissipated in the TLs connecting between the MGs and the MS due to the long distance between them. 2) The use of transformers causes a percentage of $1.5 \%$ to $2 \%$ of the transferred power to be lost. 3) At peak 
times, the main grid suffers to cover the needs of the network. 4) Blackouts can affect massive parts of the system.

In this regard, there is a pressing need to construct cooperative alliances among the MGs. This cooperative manner has plenty of benefits. As the focus is direct towards the amount of power losses, the cooperative behavior of the MGs causes a significant reduction in the amount of power dissipation. This reduction is due to three reasons. These are: 1) The short distance between the MGs, 2) As the voltage level of MGs is the same, the power is transmitted among them with no need to use a transformer, and 3) The power needs of any MG is divided among a number of sellers decreasing the amount of power flowing through any line. According to equation (2), the less the power transmitted, the less the power losses.

Cooperative Games is the branch of GT which is dedicated to study the cooperative behavior of a number of players [20]. In our case, the players are the MGs seeking to increase their pay-off by the means of reducing the losses. A transferable utility coalition game is represented by the ordered pair $(\mathcal{N}, \mathcal{V})$ where $\mathcal{N}$ denotes the set of players and $\mathcal{V}: 2^{\mathcal{N}} \rightarrow \mathfrak{R}$ with $\mathcal{V}(\phi)=0$ is the utility mapping that assigns to each coalition $S \subseteq \mathcal{N}$ a real number representing it's utility [21]. The coalitional game is characterized by the utility function $\mathcal{V}$, therefore $\mathcal{V}$ should be properly formulated for each coalition $\mathrm{S}$. Depending on the proposed algorithm, each coalition could be represented as a tripartite graph $K(n, 1, m)$ where $n=\left|S_{s}\right|$ and $m=\left|S_{b}\right|$ represent the number of MGs with surplus power and the cardinality of the set of MGs with power deficit within coalition S, respectively. 1 denotes the MS. The only station that can participate at various coalitions at the same time is the MS, therefore $\forall S_{i}, S_{j} \in 2^{\mathcal{N}}, i \neq j$ :

$$
S_{i} \cap S_{j}=\left\{\begin{array}{lr}
M S & \text { if MS } \in S_{i} \text { and } M S \in S_{j} \\
\varphi & \text { otherwise }
\end{array},\right.
$$

Within each coalition, power trade is coordinated among seller MGs, buyer MGs and the MS. The structure of coalition is shown in Figure 4. Therefore, the amount of power losses within coalition $S$ in cooperative case could be calculated as:

$$
\mathrm{P}_{\mathrm{S}}^{\text {loss }}=\left(\sum_{\mathrm{n} \in \mathrm{S}_{\mathrm{s}}, \mathrm{m} \in \mathrm{S}_{\mathrm{b}}} \mathrm{P}_{\mathrm{nm}}^{\text {loss }}+\sum_{\mathrm{n} \in \mathrm{S}_{\mathrm{s}}} \mathrm{P}_{\mathrm{n}-\mathrm{MS}}^{\text {losses }}+\sum_{\mathrm{m} \in \mathrm{S}_{\mathrm{b}}} \mathrm{P}_{\mathrm{m}-\mathrm{MS}}^{\text {losses }}\right),
$$

where $\mathrm{P}_{\mathrm{n}-\mathrm{MS}}^{\text {losses }}$, and $\mathrm{P}_{\mathrm{m}-\mathrm{MS}}^{\text {losses }}$ represent the amount of losses due to selling or purchasing power to or from the MS, respectively. Therefore, these values could be calculated directly using equation (2). $\mathrm{P}_{\mathrm{nm}}^{\text {loss }}$ denotes the amount of losses incurred in the TL when power is transmitted locally among the MGs only. $\mathrm{P}_{\mathrm{nm}}^{\text {loss }}$ is calculated using equation (2) with transformer losses $\beta=0$ and with low voltage level instead of medium voltage level used when trading power with the MS. The aim of cooperation is to minimize the amount of losses, thus:

$$
\mathrm{F}(\mathrm{S})=\min \left(\sum_{\mathrm{n} \in \mathrm{S}_{\mathrm{s}}, \mathrm{m} \in \mathrm{S}_{\mathrm{b}}} \mathrm{P}_{\mathrm{nm}}^{\text {loss }}+\sum_{\mathrm{n} \in \mathrm{S}_{\mathrm{s}}} \mathrm{P}_{\mathrm{n}-\mathrm{MS}}^{\text {losses }}+\sum_{\mathrm{m} \in \mathrm{S}_{\mathrm{b}}} \mathrm{P}_{\mathrm{m}-\mathrm{MS}}^{\text {losses }}\right),
$$

Converting the problem to a maximization problem gives the utility function $\mathcal{V}$ as:

$$
\mathcal{V}(\mathrm{S})=\max -\left(\sum_{\mathrm{n} \in \mathrm{S}_{\mathrm{s}}, \mathrm{m} \in \mathrm{S}_{\mathrm{b}}} \mathrm{P}_{\mathrm{nm}}^{\text {loss }}+\sum_{\mathrm{n} \in \mathrm{S}_{\mathrm{s}}} \mathrm{P}_{\mathrm{n}-\mathrm{MS}}^{\text {losses }}+\sum_{\mathrm{m} \in \mathrm{S}_{\mathrm{b}}} \mathrm{P}_{\mathrm{m}-\mathrm{MS}}^{\text {losses }}\right),
$$




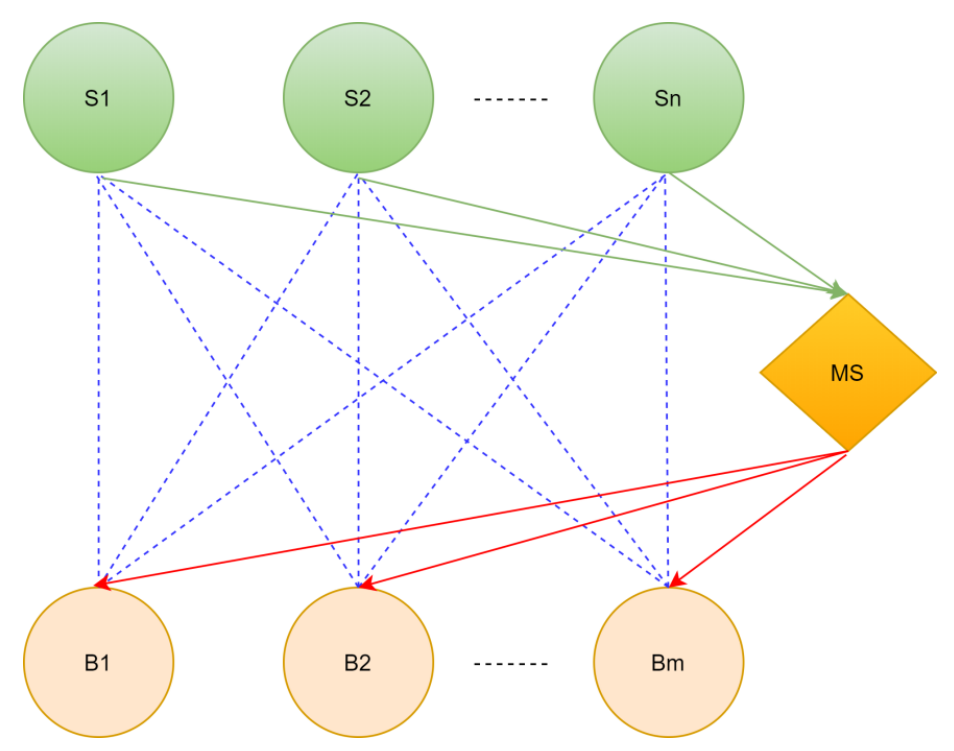

Figure 4. General structure of coalition.

The power of cooperative games appears not only in alliance construction and utility calculation of each coalition, but also in profit apportionment among members of coalitions. In transferable utility coalition games, the total utility of each coalition could be allocated among the players using various methods, including: the nucleus, the Shapley value and the fair division. The aim of this article is maximizing the utility of each formed coalition besides the problem of forming the coalitions. Therefore, proportional utility fair division is adopted to calculate the payoff of each player as follows:

$$
\phi_{\mathrm{i}}=\frac{\mathrm{v}(\{\mathrm{i}\})}{\sum_{\mathrm{j} \in \mathrm{S}} \mathrm{v}(\{\mathrm{j}\})} \mathrm{v}(\mathrm{S}),
$$

where $\mathrm{v}(\{\mathrm{i}\})$ is the non-cooperative payoff of $\mathrm{MG}_{\mathrm{i}}, \sum_{\mathrm{j} \in \mathrm{S}} \mathrm{v}(\{\mathrm{j}\})$ represents the summation of the non-cooperative payoff of all the participants of coalition $S$ and $v(S)$ is the utility of coalition $S$ in cooperative case.

The CF algorithm and the matching problem are discussed in the following section.

\section{iv. Coalition Formation Algorithm And Matching Problem}

The cooperation among the MGs is achieved via a CF algorithm. The proposed algorithm consists of two stages. In the first step, the MGs construct stable coalitions among themselves. In the second step, the buyer MGs are matched to the seller MGs in order to reduce the losses while totally meeting the requirements of the MGs with lack of power.

Algorithm 1 shown in Figure 5, gives the pseudo code of the proposed supervised distance-based CF algorithm. The algorithm could be used in all phases of network operation, i.e. it could be used in design (start-up) phase, normal operation and updating phase. The algorithm takes a set of MGs as an input and gives a set of stable coalitions reducing the power losses as an output. The day is partitioned into periods by the system operator. At the beginning of each period, the MGCCs send the data of their consumption and load levels to the PMC. The PMC collects the data of the entire system and after that gives its instructions to MGs to start the process of forming coalitions. Only active MGs i.e. MGs with $\mathrm{P}_{\text {net }} \neq 0$ can participate in the process of CF. Initially, a number $\mathrm{k}$ equals the number of active MGs (or 
appropriate number less than $\mathrm{k}$ ) of coalitions is generated (line 1 of Algorithm 1). After that, the active MGs start to construct all possible coalition collections (starting from the grand coalition ending with a network with $\mathrm{k}$ coalitions) using a distance-based scheme (line 2-5 of Algorithm 1). After the stable coalitions are created, each coalition $S$ computes the amount of aggregate power within its borders i.e. the difference between the generated power and the loads of all MGs in S. If the aggregate power is greater than 0, the MS joins the coalition as a buyer, otherwise it joins the coalition as a seller (lines 8-12 of Algorithm 1). The next step is to match the sellers to buyers within each coalition. As the aim of the problem is to reduce the power losses, and since the power losses amount is directly proportional to the square of transmitted power as mentioned in equation (2), then QP is the best suited method for solving this optimization problem. After matching the sellers to the buyers within each coalition, the utility of each MG is calculated to make sure that all MGs have no intention to leave its coalition (lines 14 to 17 of Algorithm 1). Pareto ordering concept is used to reach the stable coalition state. Pareto order states that a collection of coalitions $\mathrm{C}$ is preferred over another collection $\mathrm{K}$, if at least one participant can increase its payoff without decreasing the utility of any other participant when switching from $\mathrm{K}$ to $\mathrm{C}$. The last step is to compare between the power losses in each scenario selecting the scenario that reduce the losses of the entire system (lines 19, 20 of Algorithm 1).

The seller-buyer matching problem is formulated as a QP problem as suggested in [10]. The reason behind that is the quadratic nature of the power losses. The amount of power losses is directly proportional to the square of power transmitted in TL coupling the buyer with the seller as mentioned before. Sequential least square quadratic programming (SLSQP) explained in [22] is used to solve the problem. The aim of the problem is to properly match the sellers to the buyers in a way to reduce the losses in addition to meeting the needs of the buyers. The problem could be formulated as in equation (9) as:

$$
\min \left(\sum_{\mathrm{n} \in \mathrm{S}_{\mathrm{s}}, \mathrm{m} \in \mathrm{S}_{\mathrm{b}}} \mathrm{P}_{\mathrm{nm}}^{\text {loss }}+\sum_{\mathrm{n} \in \mathrm{S}_{\mathrm{s}}} \mathrm{P}_{\mathrm{n}-\mathrm{MS}}^{\text {losses }}+\sum_{\mathrm{m} \in \mathrm{S}_{\mathrm{b}}} \mathrm{P}_{\mathrm{m}-\mathrm{MS}}^{\text {losses }}\right)
$$

\section{Subject to:}

Seller's feeder constraints (The power delivered from seller $\mathrm{n}$ to buyer $\mathrm{i}$ can't exceed the net power of seller $\mathrm{n}$. The seller should be able to meet the needs of the buyer in addition to the losses incurred in the TLs)

$$
\begin{gathered}
0 \leq P_{n i}^{\text {del }} \leq P_{n}^{\text {seller }}, \\
P_{n}^{\text {seller }}-P_{n i}^{\text {losses }}-x_{n i} \geq 0,
\end{gathered}
$$

Buyer's feeder constraint (The amount of power injected to buyer i should ensure that the needs of it are totally met)

$$
P_{i}^{\text {buyer }}-\sum_{n=0}^{s} P_{n i}^{\text {losses }}+\sum_{n=0}^{s} x_{n i}=0,
$$

where $\mathrm{P}_{\mathrm{i}}^{\text {buyer }}$ is the power status of buyer $\mathrm{i}$ (negative value), $\mathrm{P}_{\mathrm{ni}}^{\text {losses }}$ denotes the losses incurred in TL between seller $\mathrm{n}$ and buyer $\mathrm{i}$, and $\mathrm{x}_{\mathrm{ni}}$ is the amount of power transmitted from seller $\mathrm{n}$ to buyer $\mathrm{i}$. 


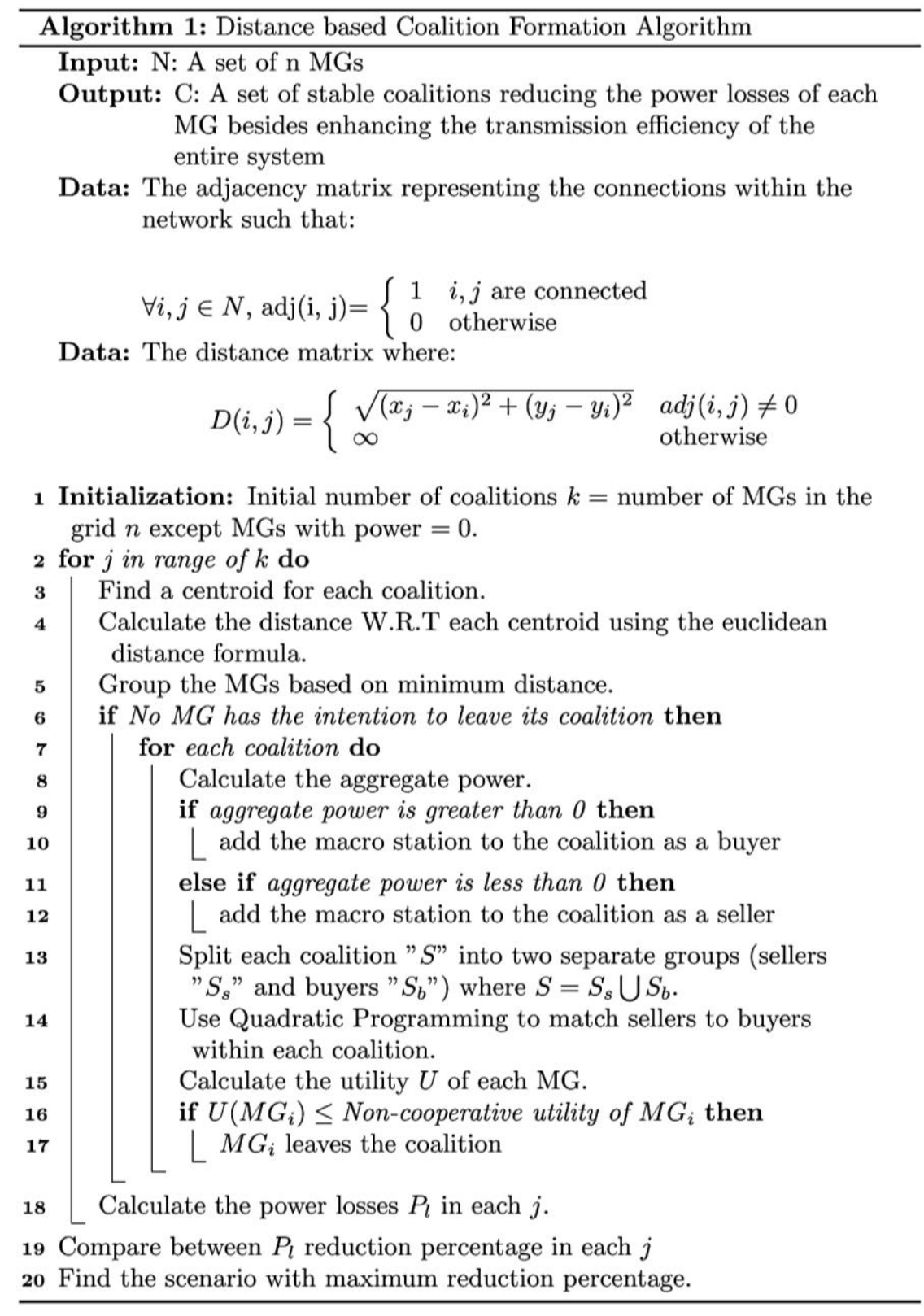

Figure 5. Supervised distance based CF algorithm

As mentioned before, a key limitation of the merge and split algorithm used in [12] is that it is NP hard which makes it not scalable [13]. On the contrary, our algorithm depends on a distance-based technique like the used in the K-means clustering algorithm in the part of the algorithm related to the process of forming coalition. Thus, the time complexity of our algorithm in the phase of coalition forming equals the time complexity of the K-means which is $O(I K N T)$ as illustrated in [24], where $I$ is the number of iterations, $K$ is the number of coalitions formed, $N$ is the number of MGs included in the game, and $T$ is the time needed to calculate the distance.

\section{RESULTS}

In this section, the performance of the presented algorithm is illustrated. For simulation, we construct a $20 \mathrm{~km} \times 20 \mathrm{~km}$ square distribution network with a MS located at the center of the network. The MS 
connected from medium voltage level side to the main grid and from the low voltage level side to a set of MGs randomly scattered within the distribution network. The medium and low voltages are set to $69 \mathrm{KV}$ and $33 \mathrm{KV}$, respectively. The power factor is assumed to equal 0.8 . The position of the MS is set to be at the origin point $(0,0)$. Analogously to [12], the net power $\mathrm{P}_{\text {net }}$ of each $\mathrm{MG}$ is considered to be a Gaussian random variable which has a mean $(\mu=0)$ and a standard deviation having a uniform distribution with a value between $10 \mathrm{MW}$ and $316 \mathrm{MW}$. The resistance per km of all TLs is set to be $0.273 \Omega / \mathrm{km}$ [23]. The transformer losses are in general between $1.5 \%$ and $2 \%$ of the transmitted power. In this work, the transformer losses value is assumed to be $1.5 \%$ and the price per MW is assumed to be $\$ 1$. The model and the algorithm were implemented in Python 3.7 using spyder open source platform IDE. All simulations were performed on MS Windows 10 Pro 64-bit operating system; the CPU used is Intel Core i7-4810 MQ running at $2.80 \mathrm{GHz} ; 8 \mathrm{~GB}$ of RAM.

\section{A. Numerical Example}

As the level of generation and power needs of each MG varies from an hour to another hour throughout the day, the process of CF is implemented in an hourly base. At the beginning of each hour, the MGCCs collects the data from their respective MGs. After that, each MGCC shares the information with other MGCCs in addition to the PMC which gives the instruction to the MGs to begin forming the groups depending on their positions and power needs. In this numerical example, the effect of the proposed algorithm on reducing the power losses of the system is demonstrated. A distribution network with 15 MGs is constructed as in Figure 6. The MGs are generated randomly. Each MG has a set of attributes, such as: the position, the power status, the non-cooperative power losses, and the percentage of the amount of power losses to the total amount of transmitted power. The attributes of the 15 MGs are shown in Table 1. The proposed algorithm is applied, and all possible coalition collections are constructed. Every time, the power losses values are compared, and the best collection is chosen. In this

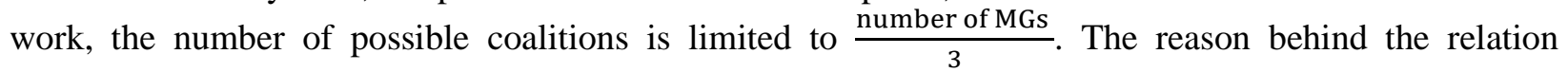
between the number of coalitions and the number of MGs is that the more the number of coalitions, the less the effect of cooperation. A comparison between the power losses in various scenarios is shown in Figure 7. The next step is to select the best coalition collection.

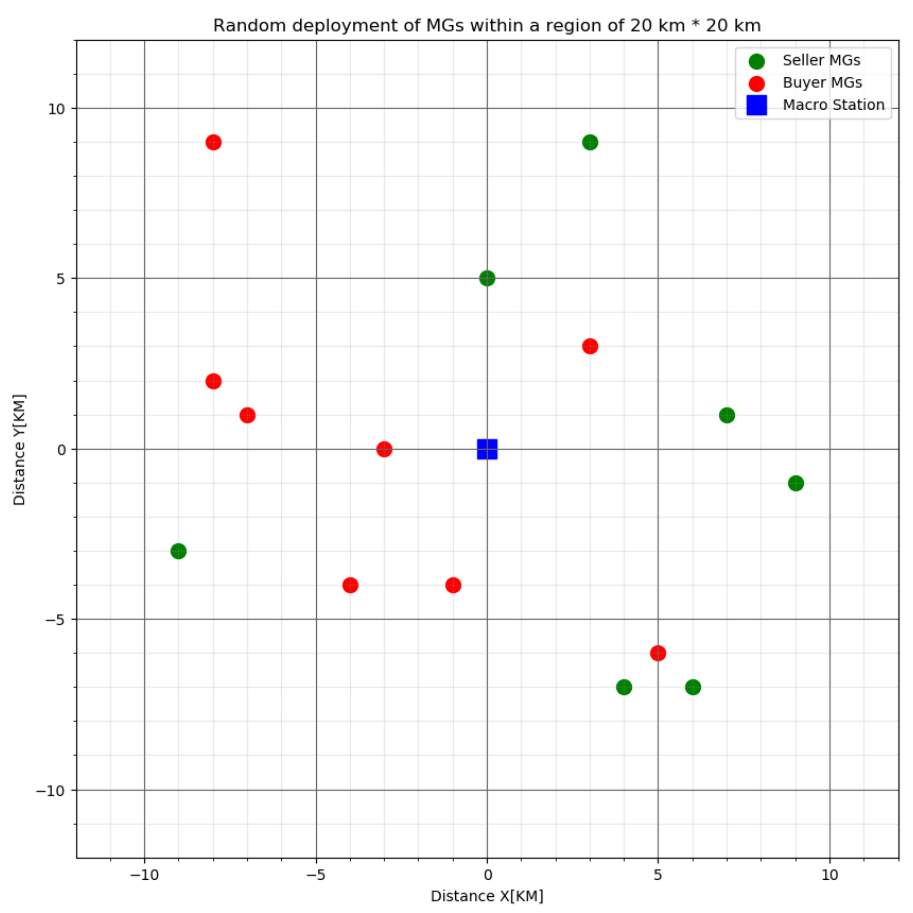

Figure 6. A distribution network with randomly scattered $15 \mathrm{MGs}$ 
Table 1 . The attributes of the MGs within the network in non-cooperative case

\begin{tabular}{|c|c|c|c|c|c|c|}
\hline \multirow{2}{*}{$\begin{array}{c}\text { MG } \\
\#\end{array}$} & \multicolumn{2}{|c|}{ Position } & \multirow{2}{*}{ Power status (Watt) } & \multicolumn{3}{|c|}{ Non-cooperative case } \\
\hline & $\mathrm{x}$ & $\mathrm{y}$ & & Power losses (Watt) & Pay off & $\frac{p_{\text {loss }}}{p_{\text {delivered }}} \%$ \\
\hline 1 & -4 & -4 & -73796642.7291158 & 4080542.7887392566 & -4080542.7887392566 & 5.52944231313834 \\
\hline 2 & 4 & -7 & 451277231.9870801 & 153874355.38177824 & -153874355.38177824 & 34.097522426343815 \\
\hline 3 & 3 & 9 & 29787629.220392488 & 1200998.9773187537 & -1200998.9773187537 & 4.031871648572001 \\
\hline 4 & 7 & 1 & 50385574.36625031 & 2364138.643234428 & -2364138.643234428 & 4.692094261047058 \\
\hline 5 & 6 & -7 & 459343592.5041991 & 181178873.64333463 & -181178873.64333463 & 39.44299574434107 \\
\hline 6 & 3 & 3 & -21239426.655147456 & 502507.8118601929 & -502507.8118601929 & 2.3659198528243146 \\
\hline 7 & -8 & 2 & -8319634.270565081 & 180008.56904577403 & -180008.56904577403 & 2.1636596416581133 \\
\hline 8 & -1 & -4 & -48571718.014520615 & 1657495.0454571862 & -1657495.0454571862 & 3.41246946414717 \\
\hline 9 & -7 & 1 & -364230945.531623 & 115438979.21359128 & -115438979.21359128 & 31.69389658671073 \\
\hline 10 & -8 & 9 & -24516926.644922387 & 1061363.7150681375 & -1061363.7150681375 & 4.32910588851459 \\
\hline 11 & 9 & -1 & 22299566.322928306 & 737938.415321696 & -737938.415321696 & 3.3092052313275317 \\
\hline 12 & 5 & -6 & -159226488.94536904 & 22790139.662765767 & -22790139.662765767 & 14.31303284630305 \\
\hline 13 & -3 & 0 & -314976469.02961963 & 34626028.69800656 & -34626028.69800656 & 10.9932112721571 \\
\hline 14 & 0 & 5 & 507871508.562875 & 123166009.46929689 & -123166009.46929689 & 24.25141150717826 \\
\hline 15 & -9 & -3 & 3467552.336944358 & 62233.30623338738 & -62233.30623338738 & 1.79473300432512 \\
\hline
\end{tabular}

In this example, the best scenario is the scenario obtained when the number of coalitions is 4 . The reason behind that is as shown in figure 7 , the amount of power losses in the case of partitioning the system into 4 coalitions is 422282632.47020006 Watt which is less than the power dissipated in all other scenarios. The final optimal grouped network is illustrated in Figure 8. Table 2 shows the members of each coalition, the utility value and the amount of power losses related to each coalition. As stated before, the proportional fair division method is used to distribute the utility among the members of each coalition. For example, the coalition $S_{2}=\{2,5,12\}$ has a utility value $v\left(S_{2}\right)=-237181754.2170607$ as stated in Table 2. The non-cooperative payoff of MG 2 is $\phi_{2}(\{2\})=-153874355.38177824$ as illustrated in Table 1. Thus, by using the fair division method, the cooperative payoff of MG 2 is

$$
\phi_{2}\left(\mathrm{~S}_{2}\right)=\frac{-153874355.38177824}{-153874355.38177824-181178873.64333463-22790139.662765767}
$$

which equals -101989285.62597625 . Table 3. shows the payoff and the percentage of losses to transmitted power in cooperative case. 


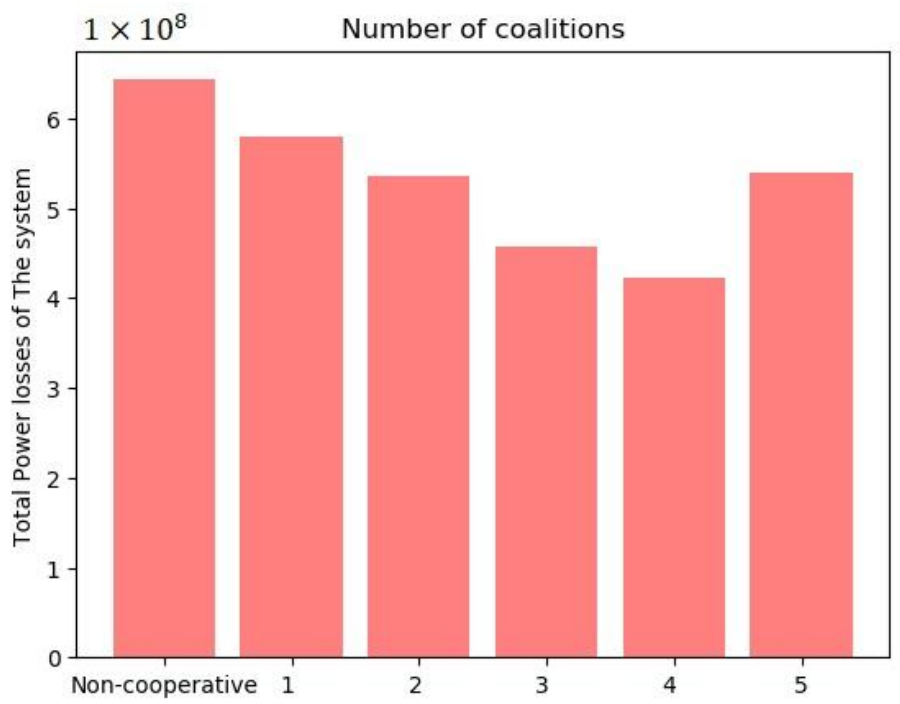

Figure 7. A comparison between the power losses (Watt) of the system in various coalition collections

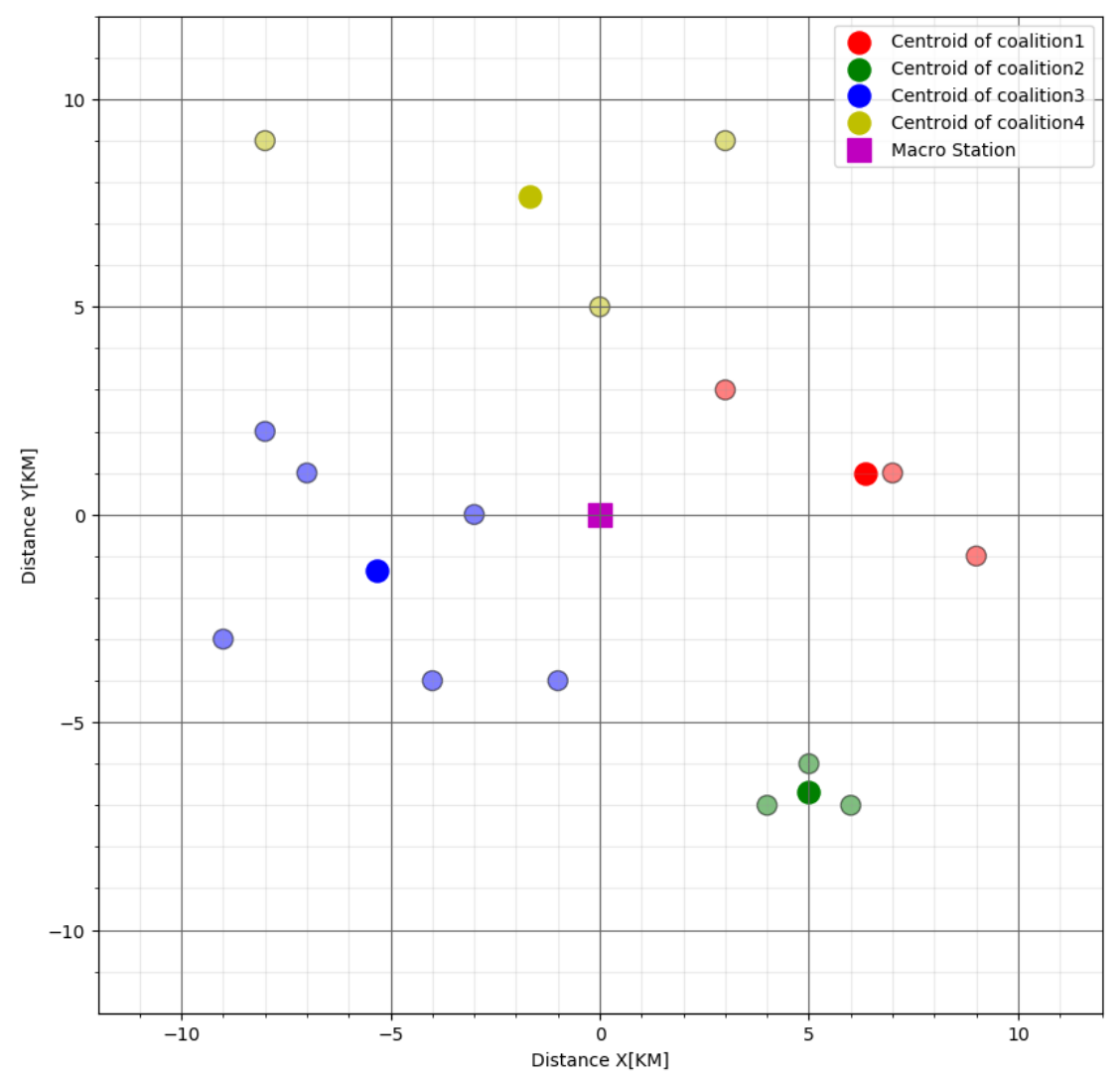

Figure 8. The coalition collection with the best performance 
Table 2 . The members and utility values of each coalition in the selected collection

\begin{tabular}{|c|c|c|c|}
\hline Coalition number & Members & Utility value & Power losses \\
\hline 1 & $4,6,11$ & -2954962.8345763218 & 2954962.8345763218 \\
\hline 2 & $2,5,12$ & -237181754.21706071 & 237181754.21706071 \\
\hline 3 & $1,7,8,9,13,15$ & -57498174.95623574 & 57498174.95623574 \\
\hline 4 & $3,10,14$ & -124647740.46232727 & 124647740.46232727 \\
\hline
\end{tabular}

Table 3. Cooperative case payoffs

\begin{tabular}{|c|c|c|c|c|c|c|}
\hline \multirow{2}{*}{$\begin{array}{c}\text { MG } \\
\#\end{array}$} & \multicolumn{2}{|c|}{ Position } & \multirow{2}{*}{ Power status } & \multicolumn{3}{|c|}{ Cooperative case } \\
\hline & $\mathrm{x}$ & $\mathrm{y}$ & & Coalition \# & Pay off & $\frac{p_{\text {loss }}}{p_{\text {delivered }}} \%$ \\
\hline 1 & -4 & -4 & -73796642.7291158 & 3 & -1503561.9899850835 & 2.0374395560299203 \\
\hline 2 & 4 & -7 & 451277231.9870801 & 2 & -101989285.62597625 & 22.60013986898771 \\
\hline 3 & 3 & 9 & 29787629.220392488 & 4 & -1193524.28992202 & 4.006778388073053 \\
\hline 4 & 7 & 1 & 50385574.36625031 & 1 & -1938071.1171149574 & 3.8464801512972984 \\
\hline 5 & 6 & -7 & 459343592.5041991 & 2 & -120086962.16829716 & 26.14316692949176 \\
\hline 6 & 3 & 3 & -21239426.655147456 & 1 & -411945.3311581038 & 1.9395313152592435 \\
\hline 7 & -8 & 2 & -8319634.270565081 & 3 & -66327.95103527239 & 0.7972459951748251 \\
\hline 8 & -1 & -4 & -48571718.014520615 & 3 & -610738.9820333212 & 1.2573962935606673 \\
\hline 9 & -7 & 1 & -364230945.531623 & 3 & -42535924.825300224 & 11.67828416204883 \\
\hline 10 & -8 & 9 & -24516926.644922387 & 4 & -1054758.0791482122 & 4.3021627238365925 \\
\hline 11 & 9 & -1 & 22299566.322928306 & 1 & -604946.3863032605 & 2.712816821380322 \\
\hline 12 & 5 & -6 & -159226488.94536904 & 2 & -15105506.422787309 & 9.486804942342252 \\
\hline 13 & -3 & 0 & -314976469.02961963 & 3 & -12758690.034602182 & 4.050680380635795 \\
\hline 14 & 0 & 5 & 507871508.562875 & 4 & -122399458.09325704 & 24.100477390356282 \\
\hline 15 & -9 & -3 & 3467552.336944358 & 3 & -22931.17327965414 & 0.6613072003366306 \\
\hline
\end{tabular}

The results summarized in Table 4. show an individual improvement in the payoff reaching up to $63.15 \%$ when compared to the non-cooperative case. The results show that the proposed algorithm has reduced the overall losses of the system from 642921613.3410523 to 422282632.47020006 with an improvement with a percentage of $34.31 \%$ as shown in Figure 9. (a). Figure 9. (b) illustrates that average power losses per bus has also decreased from 40182600.83381577 to 26392664.529387504 with the same percentage of improvement. The efficiency of transmission is calculated using the formula: 


$$
\eta=\frac{P_{\text {recieved }}}{P_{\text {sent }}}=\frac{P_{\text {sent }}-P_{\text {losses }}}{P_{\text {sent }}}=1-\frac{P_{\text {losses }}}{P_{\text {sent }}},
$$

The simulation results illustrate that the percentage of losses to total power is reduces from $25.3 \%$ in the non-cooperative case to $16.6 \%$ in the cooperative case. Thus, the transmission efficiency increases from $74.68 \%$ to $83.37 \%$ with a percentage of improvement equals $11.63 \%$ as shown in Figure 10.

Table 4. Individual payoff evolution

\begin{tabular}{|c|c|c|c|}
\hline $\begin{array}{c}\text { MG } \\
\#\end{array}$ & Non-cooperative Pay off & Cooperative Pay off & Improvement\% \\
\hline 1 & -4080542.7887392566 & -1503561.9899850835 & 63.15289245013331 \\
\hline 2 & -153874355.38177824 & -101989285.62597625 & 33.71911429105242 \\
\hline 3 & -1200998.9773187537 & -1193524.28992202 & 0.6223725030491778 \\
\hline 4 & -2364138.643234428 & -1938071.1171149574 & 18.02210404786406 \\
\hline 5 & -181178873.64333463 & -120086962.16829716 & 33.719114291052435 \\
\hline 6 & -502507.8118601929 & -411945.3311581038 & 18.02210404786409 \\
\hline 7 & -180008.56904577403 & -66327.95103527239 & 63.152892450133315 \\
\hline 8 & -1657495.0454571862 & -610738.9820333212 & 63.15289245013331 \\
\hline 9 & -115438979.21359128 & -42535924.825300224 & 63.152892450133315 \\
\hline 10 & -1061363.7150681375 & -1054758.0791482122 & 0.6223725030491778 \\
\hline 11 & -737938.415321696 & -604946.3863032605 & 18.02210404786409 \\
\hline 12 & -22790139.662765767 & -15105506.422787309 & 33.71911429105242 \\
\hline 13 & -34626028.69800656 & -12758690.034602182 & 63.152892450133315 \\
\hline 14 & -123166009.46929689 & -122399458.09325704 & 0.6223725030491778 \\
\hline 15 & -62233.30623338738 & -22931.17327965414 & 63.15289245013331 \\
\hline
\end{tabular}

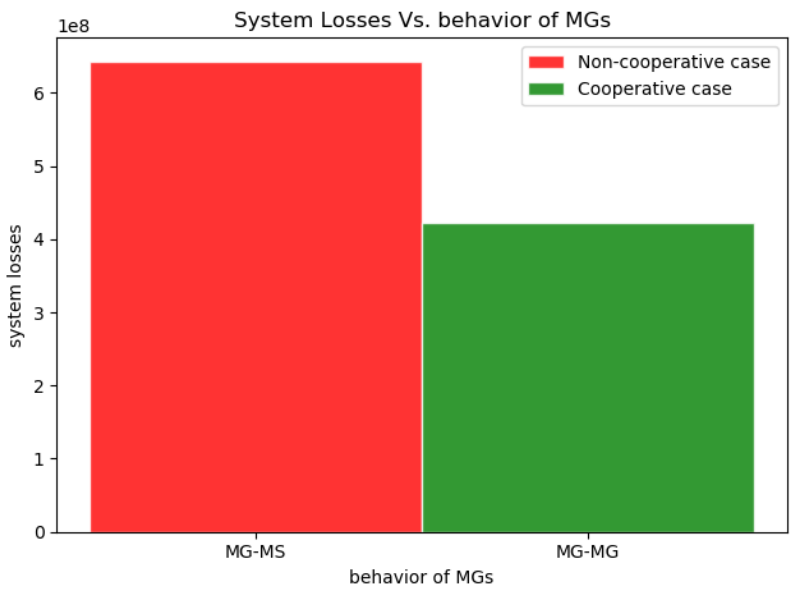

(a)

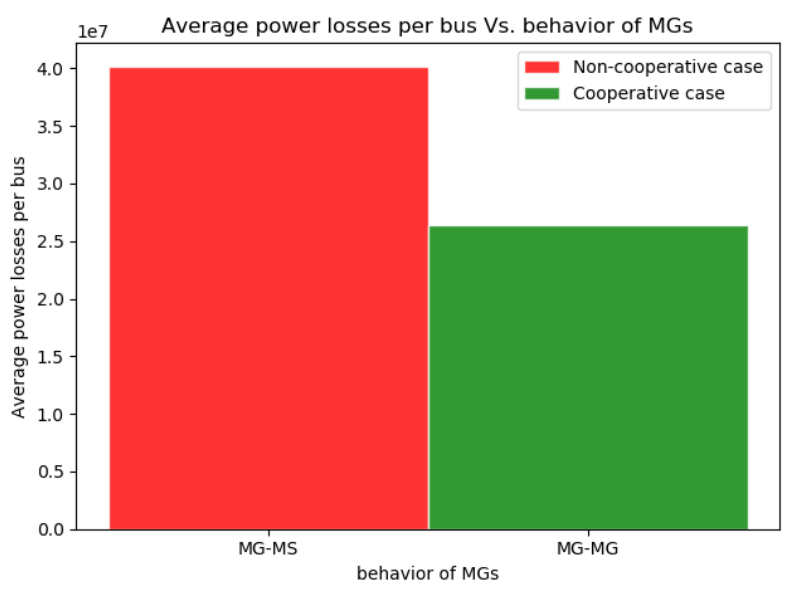

(b)

Figure 9. Comparison between the amount of power losses in cooperative and non-cooperative case 


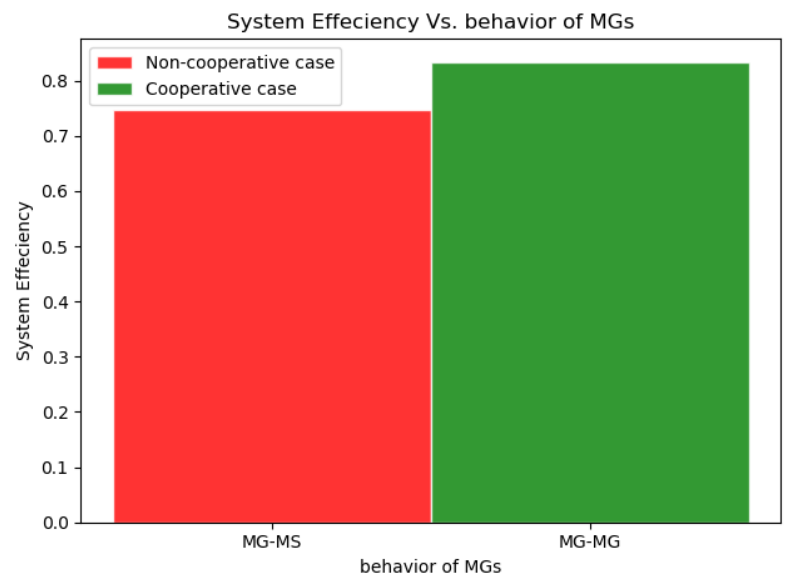

Figure 10. A comparison between the transmission efficiency in cooperative and non-cooperative case

\section{B. 500-Run Simulation}

To validate the results of the algorithm, a 500 run simulation with a variable number of MGs ranging from 2 to 32 has been performed. Figure 11. shows a significant reduction in the amount of power losses in the case of cooperative MGs when compared with the non-cooperative behavior of the MGs. The average power loss per MG is decreased in the case of $2 \mathrm{MGs}$ from 19.7 MW to $16.6 \mathrm{MW}$ with a percentage of improvement reaching 15.6\%. It is obvious from Figure 11 and Figure 12 that the more the MGs the more the power reduction. This is due to two reasons. 1) With a large number of MGs, the amount of power transmitted is distributed among a larger number of MGs decreasing the power transmitted per line, consequently decreasing the power losses per line. 2) With a large number of MGs, a MG can find easily another MG to trade power with. Figure 12. illustrates that the percentage of improvement increases with increasing the number of MGs reaching 37.61\% in the case of running with 32 MGs.

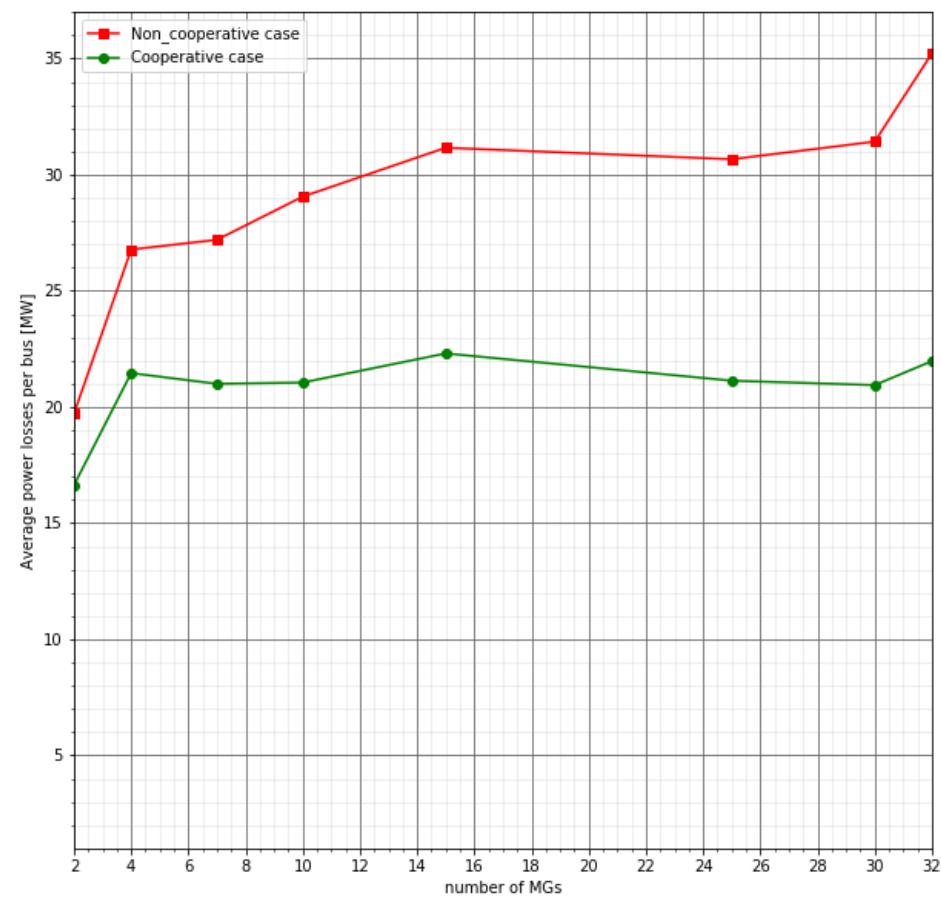

Figure 11. Evaluation of the performance of the proposed algorithm with a varying number of MGs 


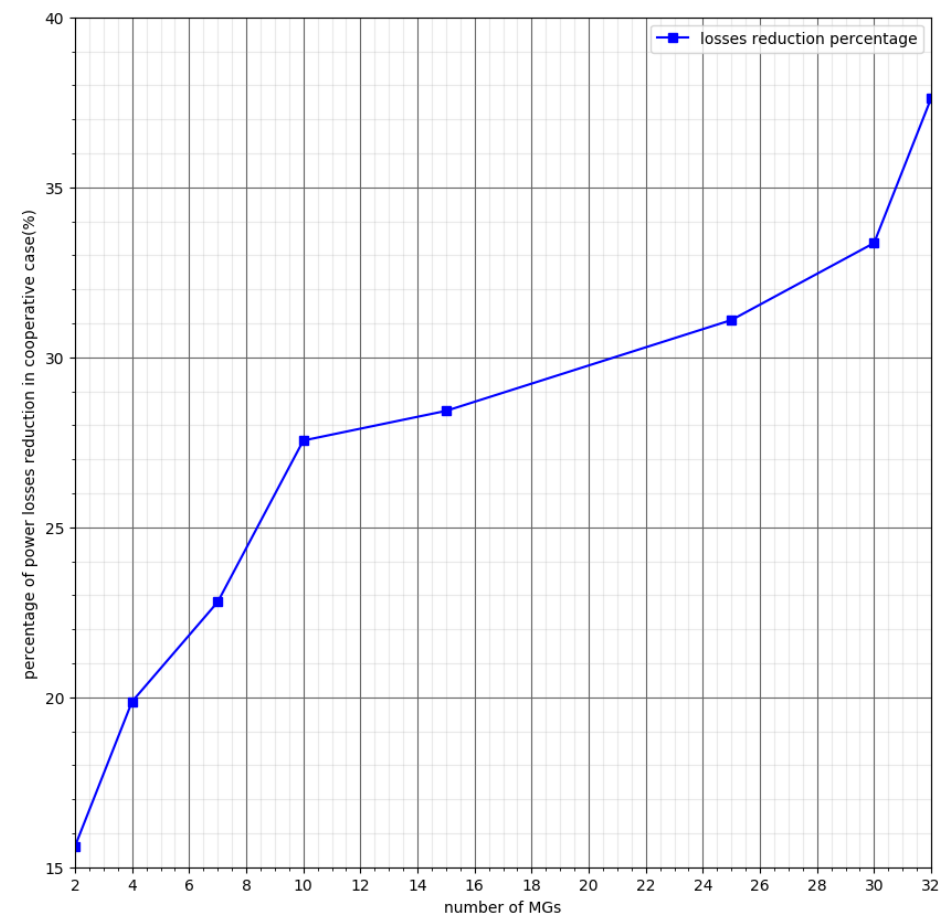

Figure 12. Percentage of losses reduction improvement when implementing the proposed algorithm

\section{CONCLUSIONS}

In this paper, we have introduced a supervised distance-based CF algorithm. The proposed algorithm allows the MGs within the distribution network to form coalitions. The aim of the CF is to exchange the power locally in a way to reduce the power losses. The main contribution of the paper consists of: 1) Introducing a scalable coalition forming algorithm, and 2) Solving the seller-buyer matching problem within each coalition. The proposed approach is described to be scalable due to the fact that it has a low time complexity. A quadratic programming problem was formulated to match the sellers to buyers in a proper way to reduce the losses within each coalition. The results obtained show that the proposed algorithm has a considerable impact on the amount of power dissipated in the system. It has been demonstrated that the proposed algorithm achieves a significant reduction in terms of power losses which improves the transmission efficiency of the system. The findings suggest that this approach could be implemented in practical life. In our future research, we intend to focus on the effect of the absence of the main grid on the system and how the storage devices can change the construction of the power market.

\section{REFERENCES}

[1] Hassan, R. and G. Radman, "Survey on smart grid", Proceedings of the IEEE, SoutheastCon 2010 (SoutheastCon), pp. 210-213, 2010.

[2] Saad, W., et al., "Game-theoretic methods for the smart grid: An overview of microgrid systems, demand-side management, and smart grid communications", IEEE Signal Processing Magazine, Vol 29, no. 5, pp. 86-105, 2012.

[3] Dimeas, A. and N. Hatziargyriou, "A multi-agent system for microgrids", Hellenic Conference on Artificial Intelligence, Berlin, pp. 447-455, 2004. 
[4] Kroposki, B., et al., "Making microgrids work", IEEE power and energy magazine, Vol 6, no. 3, pp. 40-53, 2008.

[5] Wilsun, Xu, K. Mauch, and S. Martel., "An assessment of distributed generation islanding detection methods and issues for Canada", CANMET Energy Technology Centre-Varennes, Natural Resources Canada, Canada, Rep. no. CETC-Varennes 2004-074 (TR), 2004.

[6] Hatziargyriou, N.D., et al., "Management of microgrids in market environment"., 2005 International Conference on Future Power Systems., Amsterdam, Netherlands, pp. 1-7, 2005.

[7] Lasseter, R., et al., "Integration of Distributed Energy Resources: The MicroGrid Concept", The CERTS Microgrid Concept. 2002, Lawrence Berkeley National Lab.(LBNL), Berkeley, CA (United States), 2002.

[8] Hartono, B. and R. Setiabudy, "Review of microgrid technology", 2013 international conference on QiR, Yogyakarta, Indonesia, pp. 127-132, 2013.

[9] Saad, W., et al., "Coalitional game theory for communication networks", Ieee signal processing magazine, Vol. 26, no. 5, pp. 77-97, 2009.

[10] Abdel-Raouf, O., M. Elsisy, and E. Kelash, "A Survey of Game Theory Applications in Electrical Power Micro-Grid Systems", International Journal of Computer Applications, Vol. 177, no. 37, pp. 25-34, 2020.

[11] Fadlullah, Z.M., et al., "A survey of game theoretic approaches in smart grid", 2011 International Conference on Wireless Communications and Signal Processing (WCSP), Nanjing,China, pp. 14, 2011.

[12] Saad, W., Z. Han, and H.V. Poor., "Coalitional game theory for cooperative micro-grid distribution networks", 2011 IEEE international conference on communications workshops (ICC), Kyoto, Japan , pp. 1-5, 2011.

[13] Chakraborty, S., S. Nakamura, and T. Okabe, "Scalable and optimal coalition formation of microgrids in a distribution system", IEEE PES Innovative Smart Grid Technologies, Istanbul, Turkey, pp. 1-6, 2014.

[14] Fadlullah, Z.M. and N. Kato, "Game-theoretic coalition formulation strategy for reducing power loss in micro grids", Evolution of Smart Grids, pp. 39-51, Springer, 2015.

[15] Cabrera, J.B., et al., "Reducing Power Losses in Smart Grids with Cooperative Game Theory", Advanced Communication and Control Methods for Future Smartgrids, IntechOpen, 2019.

[16] Shams, F. and M. Tribastone, "Power Trading Coordination in Smart Grids Using Dynamic Learning and Coalitional Game Theory", International Conference on Quantitative Evaluation of Systems, Madrid, Spain, pp. 54-69, 2015.

[17] Mei, J., et al., "Coalitional game theory based local power exchange algorithm for networked microgrids", Applied Energy, Vol. 239, pp. 133-141, 2019.

[18] Weedy, B.M., et al., Electric power systems, John Wiley \& Sons, New Jersy, 2012.

[19] Anumaka, M.J.D.O.E.E.E., "Analysis of technical losses in electrical power system (Nigerian $330 \mathrm{kV}$ network as a case study)", International Journal of Recent Research and Applied Studies, Vol. 12, no. 2, pp. 320-327, 2012.

[20] Branzei, R., D. Dimitrov, and S. Tijs, Models in cooperative game theory, Springer Science \& Business Media, Berlin, 2008.

[21] Peleg, B. and P. Sudhölter, Introduction to the theory of cooperative games, Springer Science \& Business Media, Berlin, 2007.

[22] Nocedal, J. and S.J.J.N.o. Wright, "Sequential quadratic programming", Numerical Optimization, pp. 529-562, Springer Science \& Business Media, Berlin, 2006.

[23] Louie, H., Off-grid electrical systems in developing countries, Springer, Berlin, 2018.

[24] Patel, Vaishali R., and Rupa G. Mehta, "Impact of outlier removal and normalization approach in modified k-means clustering algorithm", International Journal of Computer Science Issues (IJCSI), Vol. 8, no. 5, pp. 331-336, 2011. 